
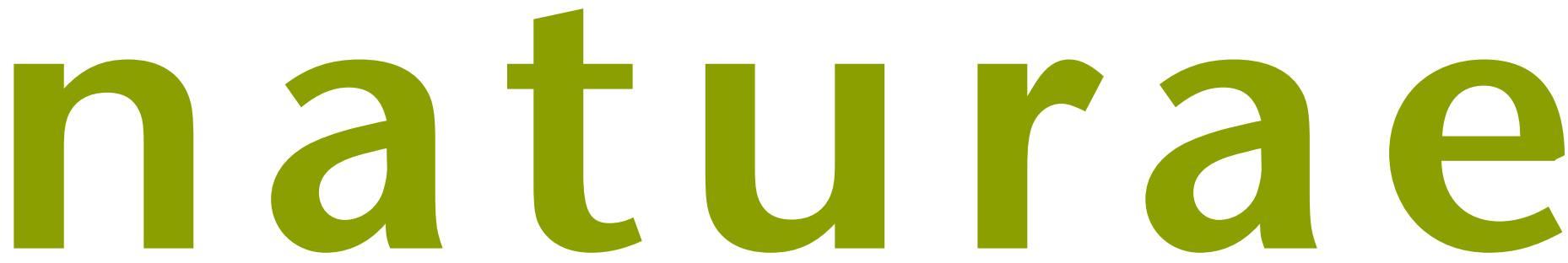

$2021 \cdot 26$

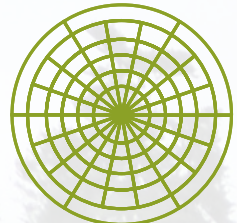

REVER 10 - 10E COLLOQUE DU RÉSEAU D'ÉCHANGE ET DE VALORISATION EN ÉCOLOGIE DE LA RESTAURATION PARIS, 19-21 MARS 2019

Édité par Philippe GOURDAIN

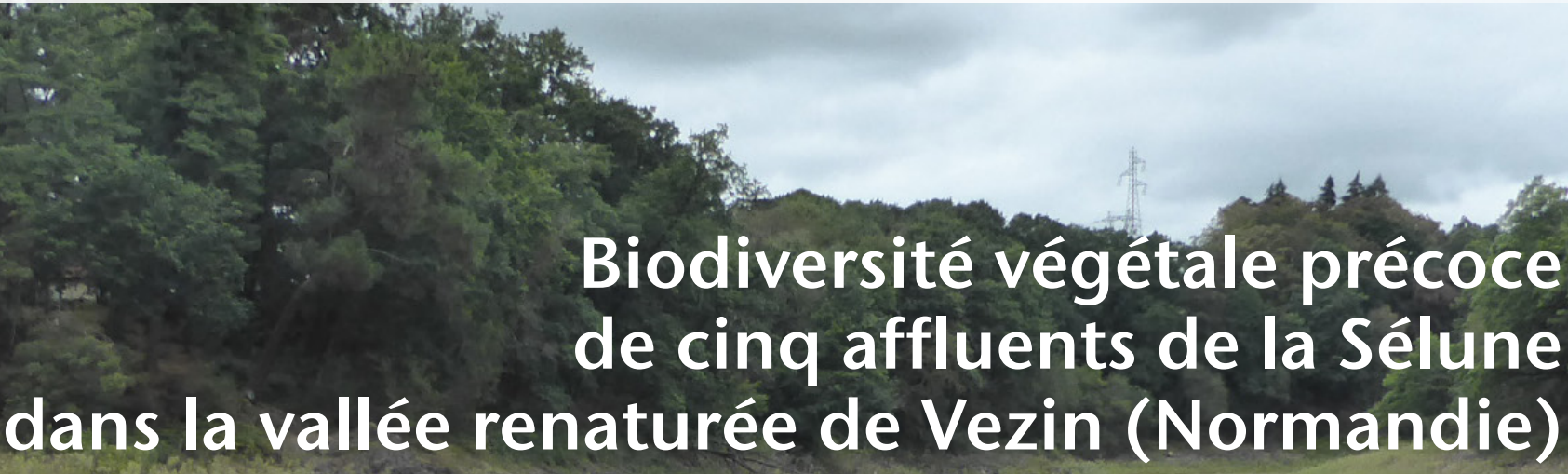

Charlotte RAVOT, Didier LE CCEUR, Simon DUFOUR \& Ivan BERNEZ

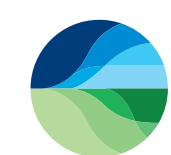

(O) OFFICE FRANCCAIS
PUBLICATIONS SCIENTIFIOUES 
DiRECTEUR DE LA PUBLICATION / PUBLICATION DIRECTOR: Bruno David,

Président du Muséum national d'Histoire naturelle

RÉDACTEUR EN CHEF / EDITOR-IN-CHIEF: Jean-Philippe Siblet

ASSISTANTE DE RÉDACTION / ASSISTANT EDITOR: Sarah Figuet (naturae@mnhn.fr)

Mise EN PAGE / PAGE LAYOUT: Sarah Figuet

COMITÉ SCIENTIFIQUE / SCIENTIFIC BOARD:

Luc Abbadie (UPMC, Paris)

Luc Barbier (Parc naturel régional des caps et marais d'Opale, Colembert)

Aurélien Besnard (CEFE, Montpellier)

Vincent Boullet (Expert indépendant flore/végétation, Frugières-le-Pin)

Hervé Brustel (École d'ingénieurs de Purpan, Toulouse)

Patrick De Wever (MNHN, Paris)

Thierry Dutoit (UMR CNRS IMBE, Avignon)

Éric Feunteun (MNHN, Dinard)

Romain Garrouste (MNHN, Paris)

Grégoire Gautier (DRAAF Occitanie, Toulouse)

Olivier Gilg (Réserves naturelles de France, Dijon)

Frédéric Gosselin (Irstea, Nogent-sur-Vernisson)

Patrick Haffner (PatriNat, Paris)

Frédéric Hendoux (MNHN, Paris)

Xavier Houard (OPIE, Guyancourt)

Isabelle Leviol (MNHN, Concarneau)

Francis Meunier (Conservatoire d'espaces naturels - Hauts-de-France, Amiens)

Serge Muller (MNHN, Paris)

Francis Olivereau (DREAL Centre, Orléans)

Laurent Poncet (PatriNat, Paris)

Nicolas Poulet (OFB, Vincennes)

Jean-Philippe Siblet (PatriNat, Paris)

Laurent Tillon (ONF, Paris)

Julien Touroult (PatriNat, Paris)

COUVERTURE / COVER:

Ruisseau de l'Isolant serpentant vers sa confluence avec la Sélune, vidange partielle en 2017. Crédit photo: Charlotte Ravot.

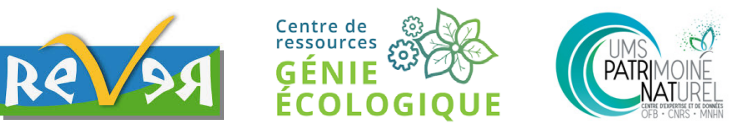

Naturae est une revue en flux continu publiée par les Publications scientifiques du Muséum, Paris

Naturae is a fast track journal published by the Museum Science Press, Paris

Les Publications scientifiques du Muséum publient aussi / The Museum Science Press also publish:

Adansonia, Zoosystema, Anthropozoologica, European Journal of Taxonomy, Geodiversitas, Cryptogamie sous-sections Algologie, Bryologie, Mycologie, Comptes Rendus Palevol.

Diffusion - Publications scientifiques Muséum national d'Histoire naturelle

CP 41 - 57 rue Cuvier F-75231 Paris cedex 05 (France)

Tél. : 33 (0)1 40794805 / Fax: 33 (0)1 40793840

diff.pub@mnhn.fr / https://sciencepress.mnhn.fr

(c) Publications scientifiques du Muséum national d'Histoire naturelle, Paris, 2021

ISSN (électronique / electronic): 1638-9387 


\title{
Biodiversité végétale précoce de cinq affluents de la Sélune dans la vallée renaturée de Vezin (Normandie)
}

\author{
Charlotte RAVOT \\ Didier LE COEUR \\ INRAE, Institut Agro, UMR Biodiversité, Agroécologie et Aménagement du Paysage (BAGAP), \\ Agrocampus Ouest, 65 rue de Saint-Brieuc, F-35042 Rennes cedex (France) \\ charlotte.ravot@ntymail.com \\ didier.lecoeur@agrocampus-ouest.fr \\ Simon DUFOUR \\ Université de Rennes 2, UMR Littoral - Environnement - Télédétection - Géomatique (LETG), \\ Place du recteur Henri Le Moal, CS 24307, F-35043 Rennes cedex (France) \\ simon.dufour@univ-rennes2.fr \\ Ivan BERNEZ \\ INRAE, Institut Agro, \\ UMR Dynamique et durabilité des écosystèmes: de la source à l'océan (DECOD), \\ Agrocampus Ouest, 65 rue de Saint-Brieuc, F-35042 Rennes cedex (France) \\ ivan.bernez@agrocampus-ouest.fr
}

Soumis le 16 décembre 2019 | Accepté le 26 juin 2020 | Publié le 15 décembre 2021

MOTS CLÉS

Écologie de la restauration, écologie des communautés végétales, mosaïque d'habitats ripariens, génie écologique.
Ravot C., Dufour S., Le Cœur D. \& Bernez I. 2021. - Biodiversité végétale précoce de cinq affluents de la Sélune dans la vallée renaturée de Vezin (Normandie), in Gourdain P. (éd.). REVER 10 - 10 e Colloque du Réseau d'Échange et de Valorisation en Écologie de la Restauration, Paris, 19-21 mars 2019. Naturae 2021 (26): 351-361. https://doi. org/10.5852/naturae2021a26

\section{RÉSUMÉ}

La restauration de la continuité écologique du fleuve Sélune se traduit par un aménagement actif correspondant à l'arasement de deux grands barrages hydroélectriques (hauteurs de $36 \mathrm{~m}$ et $16 \mathrm{~m}$ ) sans précédent en Europe. Le programme scientifique comprend une analyse de la recolonisation spontanée de l'ancien lac de Vezin (une longueur de $19 \mathrm{~km}$ pour une superficie de $160 \mathrm{ha}$ ). Des baisses de niveaux successives ont dénoyé progressivement les plages de sédiments de la néo-vallée (2015-2018). Un creusement du lit mineur, un stockage des sédiments par reprofilage localisé des berges (travaux de génie civil) ont permis la prévention du risque érosif (emportement potentiel de $700000 \mathrm{~m}^{3}$ de sédiments mobilisables). Ces vases exondées, que l'on veut bloquer dans la néo-vallée, constituent des milieux pionniers ouverts à la recolonisation végétale. Les deux années de suivi (2017-2018) visent à observer le patron spatial et la trajectoire des communautés végétales spontanées des points de vue structurel, écologique et taxonomique. Les communautés végétales spontanées des néo-rives du cours principal de la Sélune dans l'ancien lac de barrage $(19 \mathrm{~km} \times 2$ berges, Ordre de Strahler [OD] de 5) sont différentes de celles des cinq principaux ruisseaux affluents de la néo-vallée (de quelques centaines de mètres chacun au sein de la nouvelle vallée, $\mathrm{OD}$ de 1 à 3). Ces petits ruisseaux contribuent au recrutement d'une flore riparienne spontanée différent de celui des $38 \mathrm{~km}$ de rives du cours principal de la Sélune. Ces résultats de génie écologique renforcent la possibilité d'une restauration écologique passive des berges de la néo-vallée, avec l'assurance de l'obtention d'une mosaïque d'habitats ripariens. 


\begin{abstract}
Riparian biodiversity vegetation compared between five tributaries and the main stream of the neo-valley of the Sélune River just after big dam removal in Vezin (Normandy, France).

The restoration of the ecological continuity of the Sélune river is a management project of the French ministry of ecology that is removing two large hydroelectric dams ( $36 \mathrm{~m}$ high and $16 \mathrm{~m}$ high) and that is unprecedented in Europe. The scientific program includes an analysis of the spontaneous recolonization of the river margins of the neo-valley of Vezin after the lake disappeared (a length of $19 \mathrm{~km}$ and an area of $160 \mathrm{ha}$ ). The second downstream dam of la Roche-qui-Boit will be destroyed later, after the success of the first Vezin dam removal. The Sélune River is a $85 \mathrm{~km}$ long coastal river in Normandie (northwestern France). It flows from Saint-Cyr-du-Bailleul (elevation of $175 \mathrm{~m}$ ) to the Bay of Mont Saint-Michel and the English Channel. This low-energy river has a mean annual discharge of $10 \mathrm{~m}^{3} / \mathrm{s}$, which ranges from $3.1 \mathrm{~m}^{3} / \mathrm{s}$ in August to $20.2 \mathrm{~m}^{3} / \mathrm{s}$ in February on average. Its pluvial hydrological regime is characterized by winter floods. Its watershed of $1107 \mathrm{~km}^{2}$ mainly occurs in rural areas caracterised by intensive farming (mixed crop-livestock farming and dairy farming) and agro-industrial activities. The climate of the region is temperate and hyperoceanic with an annual mean temperature of $12{ }^{\circ} \mathrm{C}$. Although mean annual precipitations is $800 \mathrm{~mm}$, the study year was a dry one (695 $\mathrm{mm}$ in 2017). The growing season lasts from April to September. The Sélune River has been greatly impacted by its two large hydroelectric dams built in the early $20^{\text {th }}$ century: the Vezins dam located upstream (36 m high and $278 \mathrm{~m}$ wide) and the La Roche-qui-Boit dam located downstream (16 m high and $129 \mathrm{~m}$ wide). Although there was a period of conflict over the dismantling project, the dams are expected to be removed by 2019 and 2021 respectively. The Sélune River will flow continuously again, and both $25 \mathrm{~km}$ of the river channel and 181 ha of the river valley (the combined dimensions of the two reservoirs) could return to a more natural state. In the pre-removal period, a showcase engineering project focused on reservoir draining and sediment management (i.e., channel widening and in situ storage involving local-level riverbank profiling) has been conducted to avoid sediment erosion; it was a response to environmental and societal issues, as well as concerns over cultural heritage, related to the presence of the Mont Saint-Michel. The Sélune project is thus employing both a "passive hydrological" approach and an "active geomorphic" restoration method. Successive levels of the emptying of the lake of Vezin have gradually exposed the sediment beaches of the neo-valley (2015-2018). The huge civil engineering works were performed in the river bed. A storage of sediments by localized reshaping of the banks allowed the prevention of erosive risk (potential removal of $700000 \mathrm{~m}^{3}$ of mobilizable sediment). These sediments, which we want to block in the neo-valley, constitute pioneer environments opened to plant recolonization. The two years of monitoring (2017-2018) aimed to observe the spatial pattern and the trajectory of spontaneous plant communities from the structural, ecological and taxonomic points of view. The spontaneous plant communities of the neo-banks of the main course of the Sélune in the old dam lake $(19 \mathrm{~km}$ by two banks, Strahler number 5) are different from those of the five main tributary streams of the neo-valley (a few hundred meters each within the new valley, Strahler numbers from 1 to 3). These small streams contribute to the recruitment of a spontaneous riparian flora different from that of the $38 \mathrm{~km}$ of banks of the main course of Sélune. These ecological engineering results reinforce the possibility of passive ecological restoration of the banks of the neo-valley, with the assurance of ob-

taining a mosaic of riparian habitats.
\end{abstract}

\author{
Restoration ecology, \\ riparian habitats \\ dynamic \\ ecological engineering.
}

KEY WORDS

\section{INTRODUCTION}

Près de $60 \%$ des cours d'eau européens n'ont toujours pas atteint le bon état écologique malgré les efforts de renaturation engagés durant les deux dernières décennies en application de la Directive-Cadre sur l'Eau (DCE) (2000). La suppression d'obstacles en rivière est un moyen efficace, utilisé de plus en plus fréquemment, pour restaurer la continuité écologique et la qualité de l'eau (Rolan-Meynard et al. 2019). Cette pratique va s'accroître avec le vieillissement des ouvrages: en 2011, la moyenne d'âge du parc hydroélectrique français était de 63 ans et un quart des constructions avaient plus de 74 ans. Le coût des modalités d'entretien des grands barrages soumis à vérification décennale (et parfois vidange) et l'âge des bétons, peuvent accélérer le processus de démantèlement. Les constructions peuvent devenir dangereuses et ne sont plus toujours rentables (Gough et al. 2018).

La restauration écologique a pour objectif d'aider au rétablissement d'un écosystème altéré, pour le ramener vers une trajectoire ou des fonctions définies. La restauration écologique est dite "passive» quand l'intervention minimale, repose sur la suppression des sources de perturbations anthropiques en vue d'initier la régénération spontanée de l'écosystème. La restauration «active» porte sur des techniques d'ingénierie lourdes pour accélérer le processus d'autoréparation. Ces deux approches peuvent être utilisées pour revégétaliser un site à 
restaurer: soit par recolonisation végétale spontanée, soit par la réintroduction de végétaux (plantations et/ou semis). La première pratique reste peu commune (habitude interventionniste en aménagement). Elle est bien peu coûteuse et souvent couronnée de succès quand il s'agit de revégétaliser les écosystèmes ripariens au fort potentiel de régénération naturelle. Le choix initial d'un reverdissement spontané n'empêche pas des interventions ciblées visant à conserver ou régénérer un habitat donné et/ou une espèce (McDonald et al. 2016); de même, un aménagement actif peut être suivi de pratiques de restauration écologique passive (Laurent et al. 2020).

Côté lac, les suppressions de barrages vont transformer des hectares d'écosystèmes lentiques (eaux stagnantes) en de nombreux secteurs lotiques (eaux courantes). Ces transformations affectent les communautés aquatiques mais recréent des kilomètres de berge, zone d'interface riparienne, souvent cible en restauration écologique de cours d'eau mais très peu documentée: il n'existe pas en Europe d'indicateur de la végétation riparienne en application de la DCE (Wasson 2001), comme on peut le trouver sur d'autres continents (ex. l'Indice de Qualité des Bandes riveraines [IQBR] au Canada; Saint-Jacques \& Richard 1998), mais les recherches européennes sur les zones ripariennes s'y structurent (Dufour et al. 2019). La connaissance des communautés végétales et du reverdissement spontané des berges après arasement peut contribuer au choix de la restauration écologique passive de la zone de berge. Pourtant utiles au génie écologique, ces études, s'appuyant sur la reprise spontanée de la végétation sur les rives, sont inexistantes dans le cadre de la suppression de grands barrages ( $>15 \mathrm{~m}$ de haut).

La phase pionnière de recrutement des communautés végétales est primordiale car elle influence le développement ultérieur de la succession. Dans le cas des zones ripariennes, la biodiversité végétale aura un impact sur la biodiversité animale de ces écosystèmes d'interface et de la rivière (Cavaillé et al. 2013). Aussi, ces interfaces constituent les projets principaux de restauration de cours d'eau. Les plantes, par les obstacles générés par leur présence lors des crues (rugosité) et leur ancrage racinaire, dissipent l'énergie du courant et stabilisent les rives (Corenblit et al. 2007; Vargas-Luna et al. 2018). Le focus est souvent fait sur les ligneux mais on méconnaît le rôle potentiellement structurant des espèces herbacées qui s'implantent très rapidement et réalisent le couvert végétal pionnier principal. Ces connaissances écologiques peuvent éviter des replantations massives coûteuses, qui sont plus proches d'aménagements paysagers que de renaturation et souvent trop éloignés des concepts d'écologie de la restauration (McDonald et al. 2016). Des études ont montré que des bassins versants géomorphologiquement différents vont structurer les recrutements quantitatif et qualitatif des espèces ligneuses et herbacées en berge, permettant des prédictions et/ ou d'obtenir des informations pour restaurer écologiquement ces végétations ripariennes (Engelhardt et al. 2012).

La mise en assec définitive du lac en 2018, suivie de la destruction du barrage de Vezin en 2019 permet la réapparition d'une vallée où coule un fleuve côtier et ses affluents. On y découvre environ $38 \mathrm{~km}$ de berge (cours principal) et quelques centaines de mètres de ruisseaux y confluant, la Sélune reserpente dans une vallée encaissée en "V». La comparaison des communautés végétales du cours principal (Ordre de Strahler [OD] de 5) à celle des affluents (OD de 1 à 3) permet de répondre à l'inquiétude souvent formulée en restauration écologique passive: va-t-on obtenir un recrutement peu diversifié, voire monospécifique en berge? Peut-on à partir d'observations précoces établir que l'on obtiendra une mosaïque d'habitats? Comment contribuent les petits ruisseaux à la biodiversité de la néo-vallée?

Cet article est tiré de la thématique 10e Colloque du Réseau d'Échange et de Valorisation en Écologie de la Restauration - REVER 10 (Dutoit et al. 2021) et contribue à faire qu'un aménagement majeur du génie civil (arasement de grand barrage) puisse intégrer, dans la phase de cicatrisation de la néo-vallée, des pratiques s'appuyant sur des applications en écologie de la restauration à partir de données d'écologie des communautés végétales spontanées.

\section{MATÉRIEL \& MÉTHODES}

\section{CONTEXTE \& LOCALISATION}

La Sélune est un fleuve côtier du Massif armoricain de $85 \mathrm{~km}$ de long, se jetant dans la Manche dans la baie du Mont-SaintMichel (Fig. 1A, B). Le projet de restauration de sa continuité hydromorphologique est sans précédent en Europe de par la hauteur des deux barrages hydroélectriques (dont un centenaire) qu'il concerne ( $36 \mathrm{~m}$ et $16 \mathrm{~m}$ de haut). Ces deux ouvrages empêchent la libre circulation des espèces migratrices depuis le début du $\mathrm{xx}^{\mathrm{e}}$ siècle, notamment le Saumon atlantique, Salmo salar Linnaeus, 1758, et l'Anguille européenne, Anguilla anguilla (Linnaeus, 1758), deux espèces emblématiques des petits fleuves côtiers. Avant la destruction du barrage de Vezin à l'amont (Fig. 1B, «réservoir $\mathrm{V}$ »), une vidange et une gestion sédimentaire particulière ont débuté dans le lac de retenue (19 km de long, 151 ha; Fig. 1B, C), sur quatre années (2014-2018). Le barrage de La-Roche-Qui-Boit ("LRQB», Fig. 1B), plus petit, sera démantelé ultérieurement. Il sert de zone tampon pendant les travaux sur Vezin. Les $700000 \mathrm{~m}^{3}$ de sédiments mobilisables accumulés dans le lac ont été stabilisés par de colossaux travaux de génie civil pour prévenir leur emportement en aval. Le lit mineur a ainsi été recreusé et les sédiments ont été stockés localement sur site par un talutage localisé des berges - banquette de débordement des crues biennales surmontée d'une à deux terrasses d'un à deux mètres de haut. Les nouveaux espaces dénoyés et remaniés artificiellement ou non, qui ont émergé successivement, sont ouverts à la recolonisation par la végétation spontanée.

\section{ÉCHANTILLONNAGE DES COMMUNAUTÉS VÉGÉTALES SPONTANÉES DES VASES EXONDÉES}

L'objectif d'échantillonner la végétation riparienne précoce spontanée d'une néo-vallée (inondée pendant 90 ans), en comparant ses ruisseaux affluents au cours principal, est double: quelle méthode permet de prédire une possibilité de reverdissement de la vallée par restauration écologique 


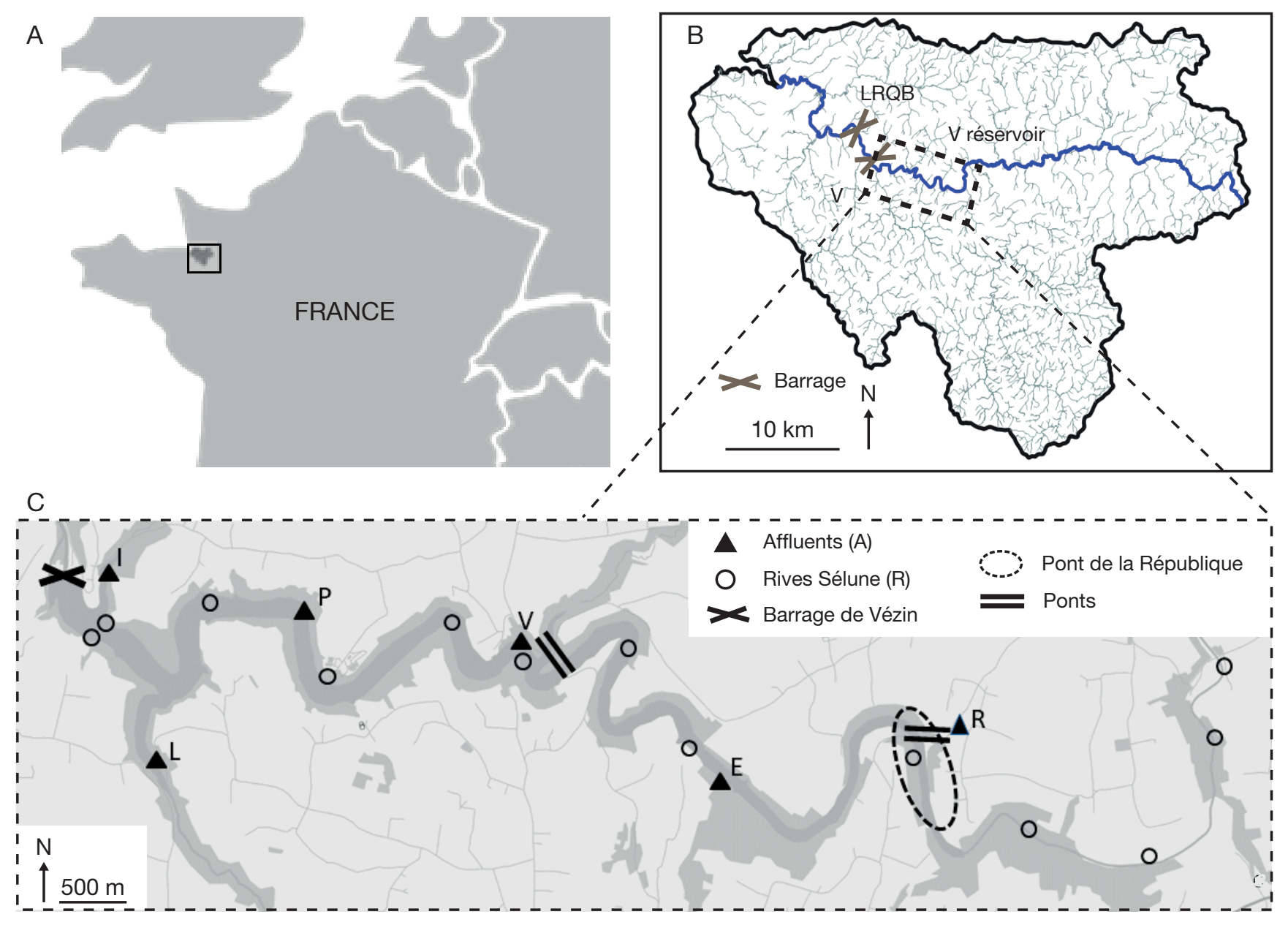

FIG. 1. - Localisation (A, B) et échantillonnage (C) des communautés végétales. Abréviations: E, ruisseau d'Esnaudières; I, ruisseau de l'Isolant; L, ruisseau du Lair; LRQB, lac de barrage de La-Roche-Qui-Boit; $\mathbf{P}$, ruisseau de Poissonnière; $\mathbf{R}$, ruisseau du pont de République; V, ruisseau de la Ville (pont des Biards). Source: contributeurs de OpenStreetMap avec l'aimable autorisation d'Andy Allan.

passive, sans plantations? Comment évaluer l'apport de biodiversité des quelques centaines de mètres de berges des principaux ruisseaux (quelques hectares pour chacun des cinq sous-bassins versants) en comparaison des 38 kilomètres de berges du cours principal (la Sélune a un bassin versant de $1083 \mathrm{~km}^{2}$, et les deux tiers au-dessus de l'ancien lac de Vezin). Cette évaluation repose sur des relevés floristiques annuels estivaux conduits sur les berges par des transects (qui forment des repères physiques) perpendiculaires au chenal. Le long de ces repères (nommés transects sur la Figure 2) des bandes de végétation homogènes $(\mathrm{U})$, définies après chaque baisse de niveau (donc dépendante du mode de gestion de la vidange) et en fonction des conditions locales, ont été délimitées (deux à cinq par transect). Dans chaque bande, trois quadrats d' $1 \times 1 \mathrm{~m}^{2}$, espacés d' $1 \mathrm{~m}$ les uns des autres, ont été placés. Les espèces végétales, leur abondance et le pourcentage de sol nu ont été relevés dans chacun des quadrats. En 2017, six stations des affluents et 13 stations du cours principal réparties sur les $38 \mathrm{~km}$ sur les berges de la Sélune, respectivement six triangles noirs et 13 cercles clairs de la Figure 1C) sont suivies. L'abaissement du lac de barrage se prolongeant au printemps 2018, permettant un meilleur accès aux berges, a fourni un échantillonnage plus aisé sur des linéaires de rives plus grands pour les affluents lors de l'été 2018. Par contre les travaux de gestion des sédiments de génie civil au pont des Biards ont obligé l'abandon d'un des affluents (affluent «Ville» noté «V» sur la Figure $1 \mathrm{C}$, il n'a été échantillonné qu'en 2017). Nous avons testé l'échantillonnage en réalisant une courbe de richesse cumulée pour l'échantillon "affluents» et l'échantillon "autres » des berges du cours principal grâce à la fonction decostand de $\mathrm{R}$ à partir du tableau des présences/ absences (méthode aléatoire, 999 permutations, ré-échantillonnage sans remise, R Core Team 2016).

Les groupes écologiques (espèces forestières, de zones humides, rudérales, prairiales) sont issus d'adaptation des classifications de Moser et al. (2002), la taxonomie suit Flora Europaea (Tutin et al. 2001).

\section{RÉSULTATS}

\section{QUEL EFFORT D'ÉCHANTILLONNAGE}

SUR DES VASES RÉCEMMENT EXONDÉES?

En 2017, 174 quadrats ont pu être ainsi suivis: 48 sur les berges des six affluents, soit six stations, et 126 sur les berges du cours principal de la Sélune, soit 13 stations réparties sur 


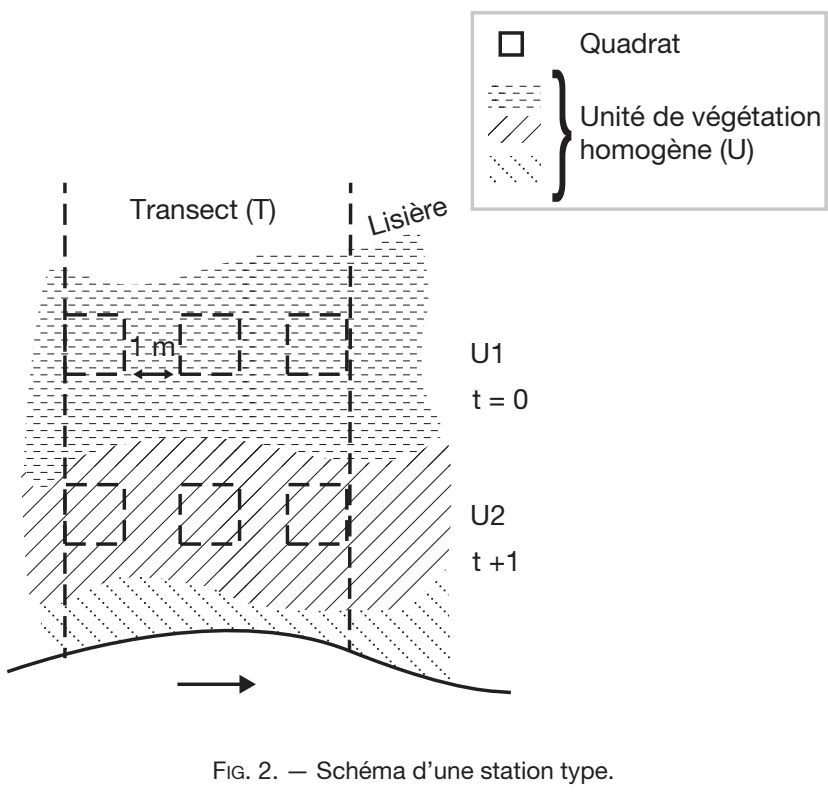

les $38 \mathrm{~km}$. La suite de la vidange et donc l'abaissement du lac de barrage se prolongeant au printemps 2018, permettant un meilleur accès aux berges, 237 quadrats ont été inventoriés: 81 sur les berges de cinq affluents (le ruisseau de la Ville [Pont des Biards] ayant dû être abandonné du fait des travaux de génie civil - gestion de sédiments) et 156 sur les berges de la Sélune.

Les courbes de richesse cumulée (Fig. 3) pour l'échantillon "Affluents" et l'échantillon "Autres" des berges du cours principal sont obtenues par la fonction decostand de $\mathrm{R}$ à partir du tableau des présences/absences (méthode aléatoire, 999 permutations, rééchantillonnage sans remise). La ligne en trait plein correspond au nombre moyen d'espèces selon l'effort d'échantillonnage et la zone grisée représente l'écart type obtenu à partir de permutations aléatoires des données. Les deux échantillons ont la même tendance, croissance qui se termine en plateau, malgré un nombre différent d'observations, rendant la comparaison possible. Une plus faible richesse spécifique est rencontrée sur les rives des affluents par rapport aux autres berges du cours principal.

\section{QUELLE VÉGÉTATION PRÉCOCE DOMINANTE POUSSE} SPONTANÉMENT SUR DES BERGES INONDÉES DEPUIS 90 ANS? Les taxons des 22 espèces les plus fréquentes (> 20 occurrences) communes aux 81 Quadrats «Affluents» et aux 156 Quadrats "Rives» de la Sélune en 2018 sont présentés en Annexe 1, ceux des 26 espèces les plus fréquentes (> 20 occurrences) communes aux 48 Quadrats "Affluents» et 126 Quadrats «Rives» de la Sélune en 2017 dans l'Annexe 2. Le tendance initiale (2017) est d'avoir des dominances des plantes de zones humides et des prairiales. L'évolution en 2018 concerne une augmentation de cinq plantes rudérales et prairiales de zone humide, dont Leerzia oryzoides (L.) Sw., protégée, en liste rouge de la Basse-Normandie (Provost 1998) et suivie par le Conservatoire botanique national (CBN) de Brest. Le lot commun de 2018 est donc toujours dominé par les
A

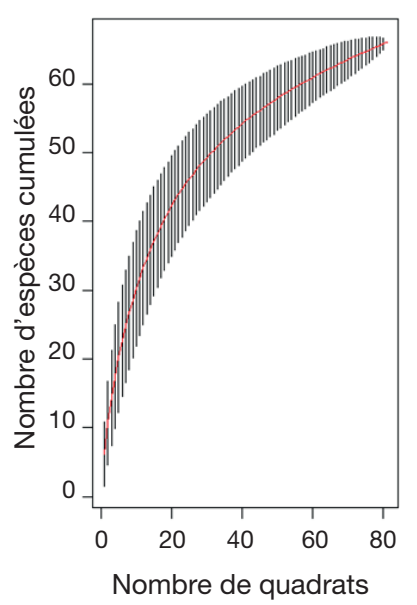

B

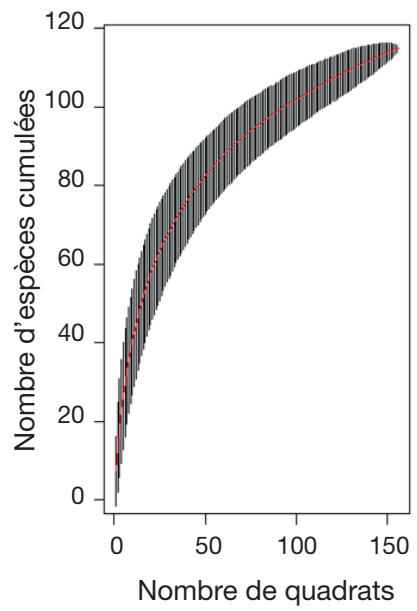

FIG. 3. - Courbes du nombre d'espèces cumulées selon l'effort d'échantillonnage sur les affluents (A) et autres sites (B) de berge du cours principal (fonction decostand de $\mathrm{R}$ à partir du tableau des Présences/Absences).

plantes herbacées et ligneuses de zone humide, avec quatre taxons de moins qu'en 2017 au-dessus des 20 occurrences, malgré l'échantillonnage facilité et augmenté. Des données semi-quantitatives de ces taxons sont présentées: les rudérales dominent; la Figure 4 donne une idée de l'évolution de la biomasse (qui n'est pas calculée: protocole trop lourd sur ces vases exondées) entre 2015 et 2018.

\section{TYPES ÉCOLOGIQUES DISTINCTIFS}

DES NÉO-BERGES DE LA SÉLUNE

Le Tableau 1 montre que le recrutement en espèces forestières diminue légèrement entre 2017 et 2018 et pour de mêmes proportions entre les rives des affluents et les rives du cours principal de la Sélune (autres sites), en proportions légèrement supérieures pour les espèces prairiales. En 2018, les berges des affluents se distinguent nettement par une baisse des espèces rudérales et une hausse forte des espèces de zone humide (> 50 \%). Sur les rives de la Sélune (autres sites 2018), les rudérales augmentent, les amphibies stagnent.

On observe une différence importante sur les plantes de zone humide (affluents > Sélune) et sur les rudérales (Sélune > affluents), des similitudes sur les espèces prairiales et forestières.

DIVERSITÉ FLORISTIQUE AU SEIN DE LA NÉO-VALLÉE

La diversité floristique entre les cinq affluents (affluents) et les autres rives du cours principal de la Sélune (autres sites) est représentée par les richesses spécifiques et les indices de Shannon en 2017 et 2018 (Fig. 5). Les différences de richesse spécifique et d'indice de Shannon ne sont plus significatifs en 2018 entre les deux échantillons de berge (affluents vs autre sites du cours principal de la Sélune), alors que la richesse et la diversité étaient significativement supérieures sur les rives des affluents en 2017. Les données d'équitabilité (Indice de Shannon ramené à une valeur maximale, 1 , où toutes les espèces de l'échantillon y étaient également représentées) sont élevées, mais la significativité statistique des résultats est faible. 

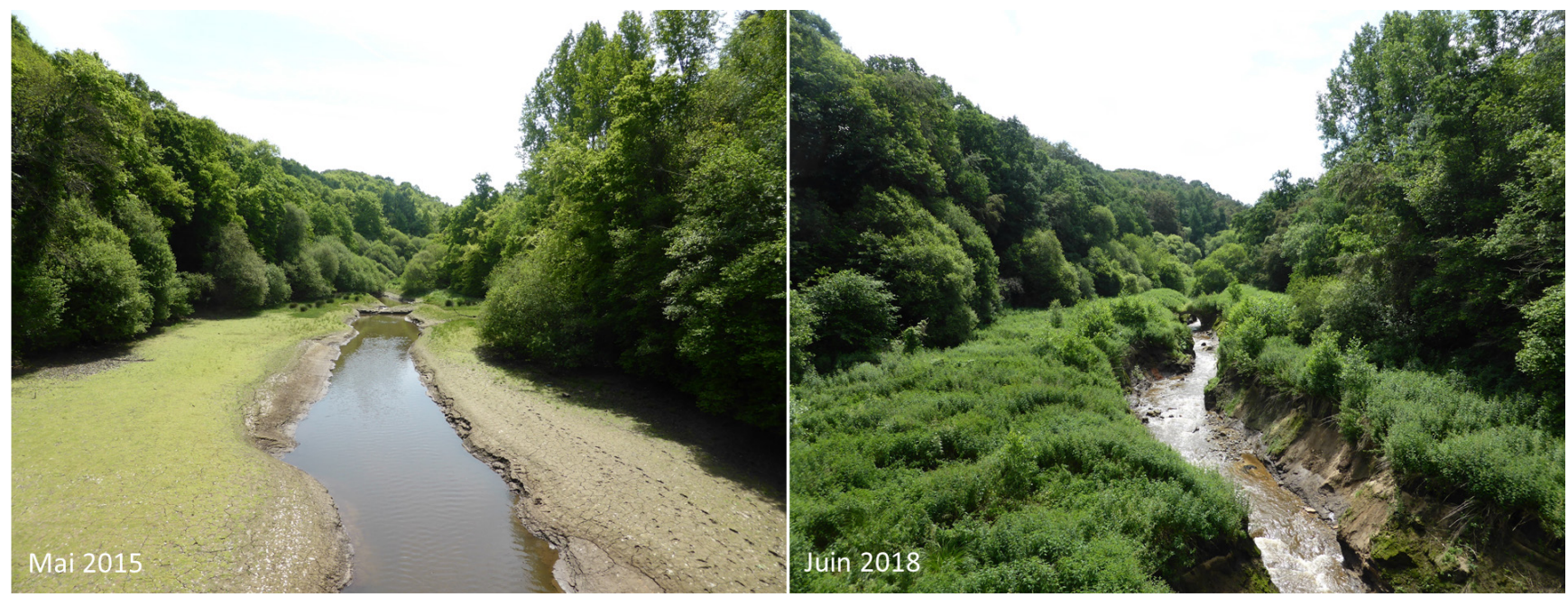

FIG. 4. - L'affluent le Lair vu depuis le cours principal de la Sélune en 2015 et 2018. Crédits photos: Aurélie Joué

TABleau 1. - Proportions de types écologiques sur les rives des ruisseaux affluents et les rives du cours principal de la Sélune sur les deux dernières années de l'assec du lac de Vezin.

\begin{tabular}{lrrrrr}
\hline & \multicolumn{2}{c}{$\mathbf{2 0 1 7}$} & & 2018 \\
\cline { 2 - 3 } \cline { 5 - 6 } Espèces & Affluents & Autres sites & Affluents & Autres sites \\
\hline forestières & $18,2 \%$ & $21,2 \%$ & & $13,6 \%$ & $15,7 \%$ \\
de zone & $39,8 \%$ & $33,0 \%$ & & $51,5 \%$ & $32,2 \%$ \\
$\quad$ humide & & & & & \\
rudérales & $30,7 \%$ & $31,4 \%$ & & $22,7 \%$ & $38,3 \%$ \\
prairiales & $7,9 \%$ & $10,2 \%$ & & $10,6 \%$ & $12,2 \%$ \\
non & $3,4 \%$ & $3,4 \%$ & & $1,5 \%$ & $1,7 \%$ \\
$\quad$ attribuées & & & & & \\
\hline
\end{tabular}

QUEL APPORT DU RECRUTEMENT SPONTANÉ PRÉCOCE EN HERBACÉES ET LIGNEUX DES CINQ AFFLUENTS COMPARÉ À CELUI DU COURS PRINCIPAL?

Les espèces les plus fréquentes (> 20 occurrences) sur les deux types de berges (affluents vs cours principal) sont les mêmes partout (Annexes 1, 2). Soixante-quatorze taxons en 2017, puis 56 en 2018 sont communs aux deux types de berge (Fig. 6). On observe des recouvrements plus importants, les deux années, sur les rives des affluents. Les cinq ruisseaux apportent chaque année une quinzaine d'espèces propres au groupe "Affluent» qu'on ne retrouve pas sur les autres rives du cours principal de la Sélune (Tableau 2). Ainsi, en 2017, une quinzaine d'espèces distinguent les affluents des rives de la Sélune (ces différences ne se maintiennent pas toutes en 2018). On constate qu'un ruisseau contribue en solo pour huit taxons spécifiques à l'échantillonnage chaque année (huit pour 14 taxons en 2017, huit pour dix taxons en 2018; Tableau 2). C'est le ruisseau de Poissonnière qui contribue le plus seul (6/16), le ruisseau de la Ville qui ne contribue jamais seul à cette spécificité des affluents par rapport aux rives du cours principal. Les autres, tel l'Isolant (Fig. 7), sont tous en intermédiaire des deux). Les taxons vus sur un ou plusieurs ruisseaux sont des plantes très classiques des zones humides et berges, ex. Amaranthus blitum L., Carex paniculata L., Carex riparia Curtis, Lamium album L., Chrysosplenium alternifolium L., Lemna minor L., Medicago lupulina L., Mentha suaveolens Ehrh., Rumex crispus L., Salix $\times$ rubens Schrank. Notons que concernant Cirsium palustre (L.) Scop., Apium nodiflorum (L.) Lag. et Stellaria alsine Grimm, qui ne sont pas non plus dans l'échantillonnage des rives du cours principal, on les trouve ailleurs dans le parcours inter-stations des rives de la Sélune. En 2018, les affluents se distinguent donc toujours par une dizaine de taxons qui peuvent eux aussi justifier de s'intéresser aux groupes écologiques de ces secteurs particuliers, typiques des rives ou ambiances fraiches et forestières (Carex paniculata, Glyceria declinata Bréb., Humulus lupulus L., Lythrum portula (L.) D.A.Webb, Typha latifolia L., Plantago lanceolata L., Scirpus sylvaticus L., Stachys sylvatica L., Geum urbanum L., Rumex obtusifolius L.). Parmi eux, les ligneux intéressent particulièrement les gestionnaires de berge. Tous les ligneux comptabilisés sur ces affluents ont été vus sur le cours principal. La richesse spécifique en ligneux est la même pour les deux années, avec douze espèces sur les rives du cours principal pour sept espèces dénombrées sur les affluents (Tableau 3): seul le ruisseau de République ne recrute qu'un taxon ligneux, les autres ruisseaux en recrutent entre trois et quatre, même sur Poissonnière qui est un ruisseau isolé, enclavé et très court. Les occurrences sont supérieures pour le saule roux et l'aulne glutineux, taxons les plus utilisés en plantation de berge dans l'Ouest.

\section{DISCUSSION}

La comparaison entre le recrutement spontané sur les rives de petits ruisseaux à celui des rives du cours principal, au sein des $19 \mathrm{~km}$ de la vallée réapparue (160 ha) suite à la vidange et la disparition du barrage de Vezin, est intéressante à plusieurs titres. L'effet d'observations à une échelle locale sur la biodiversité globale est étudié, ainsi que l'intérêt de connaître et maintenir des patchs de diversité au sein d'un paysage pour pouvoir enfin envisager une gestion à large échelle qui tende vers plus d'écologie de la restauration et moins d'aménagements actifs, polluants, coûteux et artificiels. 
A
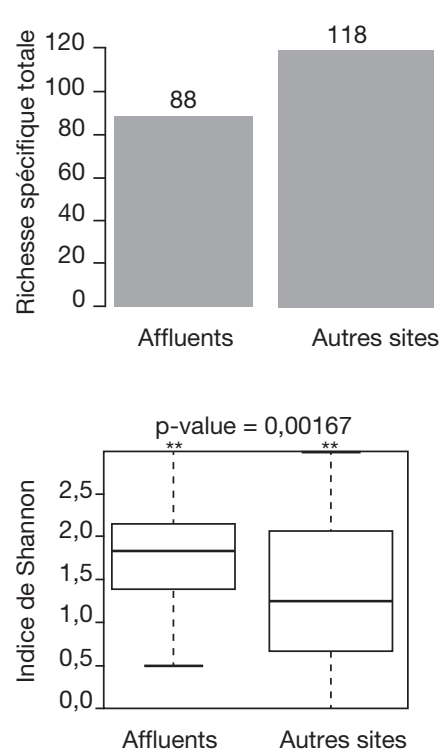
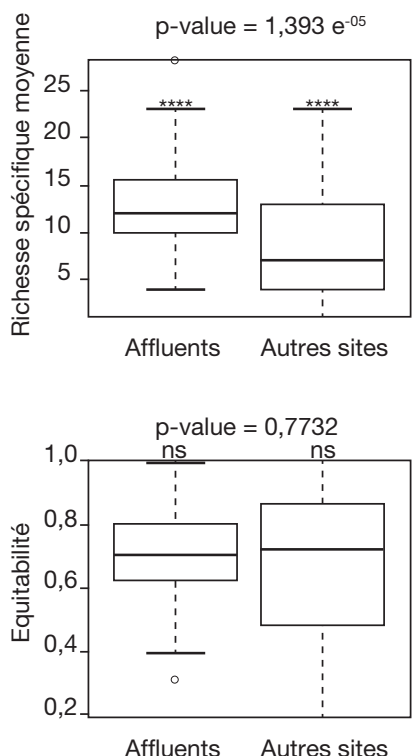

B
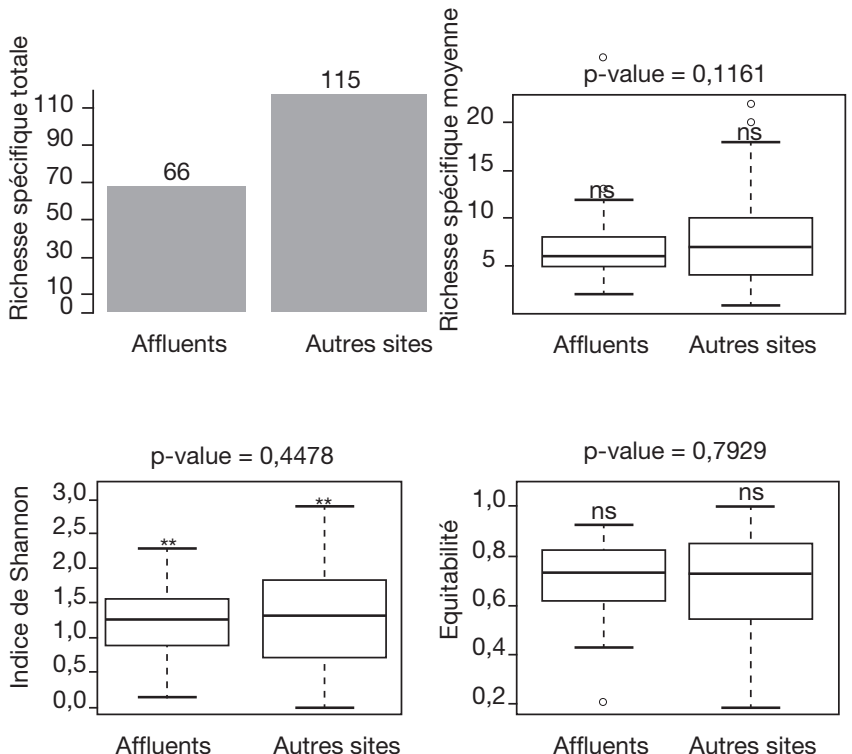

FIG. 5. - Richesse spécifique et Indices de diversité en 2017 (A) et 2018 (B) des cinq ruisseaux (affluents) et des rives du cours principal de la Sélune (autres sites).

CONTRIBUTION DE PETITS RUISSEAUX DE TÊTE

DE BASSIN VERSANT À LA BIODIVERSITÉ

D'UNE NÉO-VALLÉE D'ORDRE DE STRAHLER 5?

Concernant les ligneux, le recrutement le long du cours principal est presque deux fois supérieur (12 espèces recensées le long des rives de la Sélune). Il est intéressant d'observer cette diversité en ligneux, puisque c'est systématiquement la cible des aménagements actifs en berge (plantations d'arbres de pépinières), mais aussi la cible de la restauration écologique passive (Forget et al. 2013). Sept espèces ligneuses spontanées sont recensées précocement sur les cinq affluents: les saules et l'aulne glutineux, espèces plébiscitées en restauration de berge, faisant partie des majoritaires, alors que les surfaces d'habitats de certains affluents sont extrêmement réduites (ex. le ruisseau Poissonnière, au milieu de la néo-vallée, OD1, moins d'un kilomètre de linéaire recrute quatre des sept taxons). La viabilité des propagules observées ici après une durée d'ennoiement des sédiments sur plusieurs décades est en opposition avec d'autres suivis de recolonisation après mise en assec (Brown \& Chenoweth 2008). On peut ainsi penser que les plantes des zones humides sont mieux adaptées à des immersions longues de leur propagules. Or le recrutement des plantes de zones humides est bien supérieur (pour atteindre plus de la moitié du recrutement spontané) pour les berges des cinq ruisseaux affluents, alors qu'il ne dépasse pas un tiers pour les rives du cours principal de la Sélune. De nombreuses espèces de zones humides ou d'ambiances fraîches et forestières sont, comme nous l'avons vu, également spécifiques à ces secteurs des ruisseaux affluents et non rencontrées ailleurs sur le cours principal. Nous émettons l'hypothèse que ces cinq affluents ont une capacité d'accueil supérieure pour les plantes amphibies (effet topographique, lien avec la nappe et ambiance plus humide dans les confluences). Il est
$\mathrm{A}$

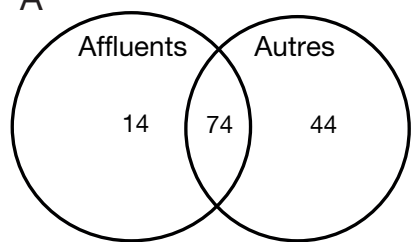

$\mathrm{B}$

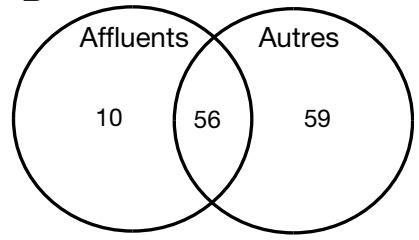

FIG. 6. - Nombres de taxons partagés et spécifiques des affluents et autres rives du cours principal en 2017 (A) et 2018 (B).

également possible qu'ils soient entretenus par des systèmes en amont: sources, zones humides, sous-bassins drainants. Alors qu'elle est très étroite, cette néo-vallée est très influencée par des apports latéraux (connectivité latérale importante pour de la biodiversité). On voit l'intérêt d'intégrer dans la gestion écologique de la vallée, la diversité floristique spécifique et le processus de recrutement important sur les rives des petits affluents.

\section{LA MOSAÏQUE D'HABITAT RIVULAIRE EN INDICATEUR D'UNE RESTAURATION ÉCOLOGIQUE RÉUSSIE?}

Alors que les linéaires de cours d'eau sont bien plus importants sur le cours principal, on n'observe pas de grande différence de richesse floristique concernant les espèces les plus abondantes: le pool commun des herbacées est assez similaire. Mais une grande différence entre les berges des affluents et les rives du cours principal va concerner le recrutement spontané des rudérales (plus fort pour les rives de la Sélune). Ravot et al. (2019) décrivent bien l'effet d'eutrophisation d'un tel système, avec des recouvrements totaux, spontanés et immédiats à l'échelle des 160 ha de la néo-vallée. L'apparition des espèces pionnières liées aux perturbations et remaniements des sédi- 
TABLEAU 2. - Taxons spécifiques en 2017 \& 2018 aux affluents de la Sélune. Abréviations: E, ruisseau d'Esnaudières; I, ruisseau de l'Isolant; L, ruisseau du lair; $\mathbf{P}$, ruisseau de Poissonnière; $\mathbf{R}$, ruisseau du pont de République; $\mathbf{V}$, ruisseau de la Ville (Pont des Biards). Si un seul quadrat est concerné, la lettre est mise entre parenthèses, par exemple: (E), vu une fois à Esnaudières.

\begin{tabular}{|c|c|c|c|}
\hline 14 taxons spécifiques aux affluents en 2017 & $\begin{array}{l}\text { Affluent(s) } \\
\text { concerné(s) }\end{array}$ & 10 taxons en 2018 & $\begin{array}{l}\text { Affluent(s) } \\
\text { concerné(s) }\end{array}$ \\
\hline Amaranthus blitum L. & $(E)$ & - & - \\
\hline Carex paniculata L. & $\mathrm{P}$ & Carex paniculata L. & $\mathrm{P}$ \\
\hline Carex riparia Curtis & LI & Geum urbanum L & $\mathrm{L}(\mathrm{l})$ \\
\hline Chrysosplenium alternifolium L. & $(P)$ & Glyceria notata Chevall. & 1 \\
\hline Cirsium palustre (L.) Scop. & P V & Humulus lupulus L. & $(\mathrm{E})$ \\
\hline Helosciadium nodiflorum (L.) W.D.J.Koch & RE I L & Lythrum portula (L.) D.A.Webb & (L) \\
\hline Lamium album L. & $(\mathrm{L})$ & Plantago lanceolata L. & $(\mathrm{R})$ \\
\hline Lemna minor L. & (P) & Rumex obtusifolius L. & (R) $\mathrm{E}$ \\
\hline Medicago lupulina L. & $\mathrm{P}$ & Scirpus sylvaticus L. & $\mathrm{P}$ \\
\hline Mentha suaveolens Ehrh. & (l) & Stachys sylvatica L. & (l) \\
\hline Ranunculus penicillatus f. terrestre (Dumort.) Bab. & $\mathrm{V}(\mathrm{E})$ & Typha latifolia L. & (l) \\
\hline Rumex crispus L. & $\mathrm{L}(\mathrm{l})$ & - & - \\
\hline Salix $\times$ rubens Schrank & $\mathrm{L}$ & - & - \\
\hline Stellaria alsine Grimm & E L V & - & - \\
\hline
\end{tabular}

ments est à mettre en lien avec la gestion active des sédiments (travaux du génie civil) qui perturbe la résilience écologique du système, visiblement très fragile (Ravot et al. 2020). Notre étude contribue à intégrer un début de connaissance sur l'apport de la biodiversité du bassin versant amont versus celle des apports latéraux: il faudrait les compléter en comparant l'effet dérive par hydrochorie, aux pluies de graines au sens large - anémochorie, zoochorie - ou l'effet d'un mixte des deux? Des différences de recrutement en plantes ripariennes entre bassins versants sont observées par ailleurs (Engelhardt et al. 2012), expliquées par des différences géomorphologiques. Il est clair que les cinq affluents (oscillants entre l'OD 1 et l'OD3) n'ont pas entre eux les mêmes morphologies et topographies, et que leurs positions, vers l'amont ou vers l'aval dans la néo-vallée, peuvent aussi avoir une influence. La meilleure connaissance des processus de colonisation dans la mosaïque de la néo-vallée permet d'envisager une gestion paysagère en écologie de la restauration, discipline trop souvent cantonnée à des espaces plus réduits.

\section{GESTION PAR LA RESTAURATION ÉCOLOGIQUE PASSIVE}

À L'ÉCHELLE PAYSAGÈRE?

Le niveau de diversité de 12 ligneux, atteint dès la fin de la vidange à l'échelle de toute la vallée échantillonnée, est intéressant. Cela augure un recrutement spontané très précoce et réparti sur toutes les berges de la vallée. Une restauration écologique passive de ripisylve est donc envisageable à un stade critique pour le gestionnaire de la néo-vallée, qui a besoin que les sédiments contenus dans l'ancien lac se stabilisent au plus vite. Par comparaison, sur un affluent de l'Oir (l'Oir étant elle-même un affluent de la Sélune), considéré comme une référence en recrutement spontané de ligneux dans une expérimentation de restauration écologique passive, ce sous bassin recrutait 13 taxons ligneux, huit ans après travaux (Forget et al. 2013). La richesse en herbacées y est également comparable: la vallée de Vezin et l'Oir amont, coulent toutes deux dans un paysage proche et subissant les mêmes pressions anthropiques. La vallée de Vezin, enclavée au niveau d'une rupture géologique, est bien dotée en ligneux, notamment sur ces versants, pour un département de la Manche qui compte très peu de forêts. Cette ambiance forestière et la présence de prairie à proximité de l'ancien lac de Vezin semble donc contribuer au recrutement sur les rives dans la néo-vallée. On observe entre les deux dates, pour tous les secteurs, une baisse globale de la richesse, alors que d'autres études montrent qu'elle croît dans les cinq premières années (Vargas-Luna et al. 2018). Mais il faudrait aussi vérifier que le reprofilage et retalutage des sédiments sur le cours principal (où des travaux du génie civil ont lieu en permanence depuis 2014 sans concertation préalable avec ceux du génie écologique) ne créent pas des conditions brutales de rupture du gradient d'humidité, avec des berges très asséchantes. C'est le cas au pont de la République sur les berges du cours principal, où la capacité de résilience des communautés de berges s'est épuisée au fil des travaux des pelleteuses (Ravot et al. 2020). Ceci expliquerait à large échelle le long des $38 \mathrm{~km}$ de berge, la recrudescence des rudérales dans ces secteurs à la défaveur des plantes d'ambiance fraîche (forestières et de zones humides) entre 2017 et 2018.

\section{CONCLUSION}

La comparaison des plantes de berges issues d'hydrosystèmes très contrastés (bassin versant de plusieurs kilomètres sur le cours principal et ordre de Strahler de 5 (OD5), et sous-bassins versants courts, d'affluents d'OD 1 à 3) permet d'envisager la restauration écologique passive comme un des outils de recolonisation et de la stabilisation des rives de la néo-vallée du lac de Vezin. Les cinq affluents contribueraient à conforter l'explication de la mosaïque d'habitats déjà observée à l'échelle de la retenue par Ravot et al. (2019), qui ne prenait pas en compte cet effet latéral. Il est intéressant de constater que de très petites surfaces d'habitat, des linéaires de rives très modestes par rapport à ceux du cours principal, vont avoir une contribution significative sur la liste d'espèces qui 


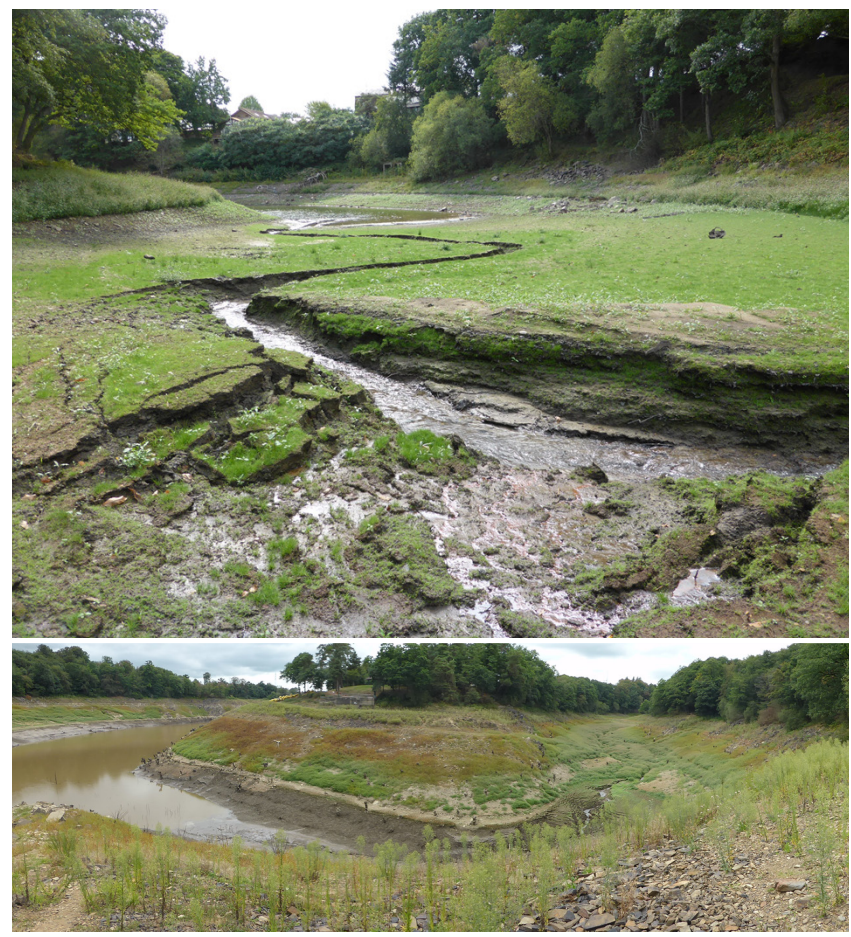

FIG. 7. - Confluence de l'Isolant avec la Sélune en 2017. Crédits photos: Charlotte Ravot.

contribueront au maintien des néo-berges. Il serait intéressant de compléter les observations taxonomiques par des études fines des traits fonctionnels (capacité de colonisation, capacité de maintien des sédiments, etc.). Il serait possible aussi d'augmenter le nombre d'affluents étudiés (un observatoire de la Sélune doit être mis en place) à l'échelle de l'ancien lac de Vezin, mais également dans le futur projet d'arasement du barrage de La-Roche-Qui-Boit, lac situé juste en aval (Fig. 1), qui comporte aussi quelques affluents courts.

\section{Remerciements}

Cette étude a été financée par l'Agence de l'Eau Seine-Normandie dans le cadre du programme SERIPAGE ( Sélune, Rives \& Paysages»). Merci aux partenaires de terrain, notamment la Direction départementale des Territoires \& de la Mer (DDTM) de la Manche, le Schéma d'Aménagement et de Gestion des Eaux (SAGE) Sélune et le CBN de Brest, antenne de Caen. Merci aux deux relecteurs et à la secrétaire de rédaction de $\mathrm{Natu-}$ rae pour l'évaluation de ce travail et les suggestions apportées.

\section{RÉFÉRENCES}

Brown R. L. \& CHENOWETH J. 2008. - The effect of Glines Canyon dam on hydrochorous seed dispersal in the Elwha River. Northwest Science 82 (sp1): 197-209. https://doi.org/10.3955/0029344X-82.S.I.197

Cavaillé P., Dommanget F., Daumergue N., Loucougaray G., Spiegelberger T., Tabacchi E. \& Evette A. 2013. - Biodiversity assessment following a naturality gradient of riverbank
TABLEAU 3. - Recrutement spontané (2017 et 2018) en ligneux sur cinq affluents de la Sélune dans l'ancien lac de Vezin. Abréviations: E, ruisseau d'Esnaudières; I, ruisseau de I'Isolant; id., pas de progression entre 2017 et 2018; L, ruisseau du lair; $\mathbf{P}$, ruisseau de Poissonnière; $\mathbf{R}$, ruisseau du pont de République; $\mathbf{T}$, tota des quadrats comportant le taxon; V, ruisseau de la Ville (Pont des Biards). Si un seul quadrat est concerné, mise entre parenthèse, par exemple: $(E)$, vu une fois à Esnaudières; >/>>, progression faible/forte entre 2017 et 2018 ; $</<<$, diminution faible/forte entre 2017 et 2018.

\begin{tabular}{|c|c|c|c|c|c|c|c|c|c|c|}
\hline \multirow[b]{2}{*}{$\begin{array}{l}\text { Ligneux des } \\
\text { affluents }\end{array}$} & \multicolumn{8}{|c|}{ AMONT > } & \multicolumn{2}{|c|}{$>$ AVAL } \\
\hline & $\mathbf{R}$ & $\mathbf{T}$ & $\mathbf{E}$ & $\begin{array}{ll}\mathbf{T} & \mathbf{l}\end{array}$ & $\mathbf{P}$ & $\begin{array}{ll}\mathbf{T} & \mathbf{l}\end{array}$ & $\mathbf{L}$ & $\mathbf{T}$ & I & $\mathbf{T}$ \\
\hline $\begin{array}{l}\text { Fraxinus } \\
\quad \text { excelsior L. }\end{array}$ & 0 & 0 & 0 & 0 & id. & 2 & 0 & 0 & 0 & 0 \\
\hline $\begin{array}{l}\text { Humulus } \\
\text { lupulus L. }\end{array}$ & 0 & 0 & id. & 3 & 0 & 0 & 0 & 0 & 0 & 0 \\
\hline $\begin{array}{l}\text { Ulex europaeus } \\
\text { L. }\end{array}$ & & 0 & 0 & 0 & $>$ & 3 & 0 & 0 & 0 & 0 \\
\hline $\begin{array}{l}\text { Salix } \times \text { rubens } \\
\text { Schrank }\end{array}$ & 0 & 0 & 0 & 0 & 0 & 0 & 0 & 2 & 0 & 0 \\
\hline $\begin{array}{l}\text { Alnus } \\
\quad \text { glutinosa } \\
\text { (L.) Gaertn. }\end{array}$ & 0 & 0 & $<$ & 1 & id. & 2 & 0 & 0 & $<<$ & 7 \\
\hline Salix alba L. & 0 & 0 & $>$ & 1 & 0 & 0 & $<$ & 1 & id. & 2 \\
\hline $\begin{array}{l}\text { Salix } \\
\text { atrocinerea } \\
\text { Brot. }\end{array}$ & $>$ & 1 & $>>$ & 5 & $>$ & 3 & id. & 14 & id. & 10 \\
\hline
\end{tabular}

protection structures in French prealps rivers. Ecological Engineering 53: 23-30. https://doi.org/10.1016/j.ecoleng.2012.12.105

Corenblit D., TABACChi E., STEIger J. \& GURnell A. M. 2007. Reciprocal interactions and adjustments between fluvial landforms and vegetation dynamics in river corridors: a review of complementary approaches. Earth Science Reviews 84 (1-2): 56-86. https://doi.org/10.1016/j.earscirev.2007.05.004

Directive-Cadre SUR L'EAU 2000. - Directive 2000/60/CE du Parlement et du Conseil établissant un cadre pour une politique communautaire dans le domaine de l'eau, 32000L0060, adoptée le 23 octobre 2000. JO du 22 décembre 2000, Luxembourg, 73 p.

Dufour S., RodríGuEZ-GONZÁlEZ P. M. \& LASLIER M. 2019. Tracing the scientific trajectory of riparian vegetation studies: main topics approaches and needs in a globally changing world. Science of the Total Environment 653: 1168-1185. https://doi. org/10.1016/j.scitotenv.2018.10.383

Dutoit T., Gallet S., Heckenroth A. \& Buisson É. 2021. 2008-2019, plus d'une décennie d'échanges et de débats autour de la restauration écologique en France, in GOURDAIN P. (éd.), REVER 10 - 10e Colloque du Réseau d'Échange et de Valorisation en Écologie de la Restauration, Paris, 19-21 mars 2019. Naturae 2021 (19): 271-276. https://doi.org/10.5852/naturae2021a19

Engelhardt B. M., Weisberg P. J. \& Chambers J. C. 2012. Influences of watershed geomorphology on extent and composition of riparian vegetation. Journal of Vegetation Science 23 (1): 127-139. https://doi.org/10.1111/j.1654-1103.2011.01328.x

Forget G., Carreau C., Le Coeur D. \& Bernez I. 2013. - Ecological restoration of headwaters in a rural landscape (Normandy, France): a passive approach taking hedge networks into account for riparian tree recruitment. Restoration Ecology 21 (1): 96-104. https://doi.org/10.1111/j.1526-100X.2012.00868.x

Gough P., Fernández Garrido P. \& VAN Herk J. 2018. — Dam Removal. A Viable Solution for the Future of our European rivers. Dam Removal Europe, 38 p.

McDonald T., Gann G. D., Jonson J. \& Dixon K. W. 2016. Standards internationaux pour la restauration écologique - incluant les principes et les concepts clés. Society for Ecological Restoration, Washington D.C, $50 \mathrm{p}$.

Laurent Y., Le Ceeur D., Rollet A.-J. \& Bernez I. 2020. - 
Évaluation précoce de la restauration écologique passive de ripisylves de ruisseaux remis dans le talweg. Techniques Sciences et Méthodes 3: 85-95. https://doi.org/10.36904/tsm/202003085

Moser D., GygaX A., BäUmler B., Wyler N. \& Palese R. 2002. Liste Rouge des fougères et plantes à fleurs menacées de Suisse. Office fédéral de l'environnement, Berne, Chambésy, Genève, 118 p.

Provost M. 1998. - Flore vasculaire de Basse-Normandie. Presse Universitaire de Caen, Tome 1, 492 p. Tome 2, 410 p.

Ravot C., Laslier M., Hubert-Moy L., Dufour S., Le Coeur D. 8 BERNEZ I. 2019. - Large dam removal and early spontaneous riparian vegetation recruitment on alluvium in a former reservoir: Lessons learned from the preremoval phase of the Sélune River project (France). River Research \& Applications 36 (6): 1-13. https://doi.org/10.1002/rra.3535

Ravot C., Laslier M., Hubert-Moy L., Dufour S., Le Coeur D. \& BERNEZ I. 2020. - Apports d'une observation précoce de la végétation spontanée pionnière pour la renaturation des rives de la rivière Sélune. Sciences Eaux \& Territoires (hors-série 62). https://doi.org/10.14758/SET-REVUE.2020.HS.02

Rolan-Meynard M., Vivier A. \& ReYjol Y. 2019. — Guide pour l'élaboration de suivis d'opérations de restauration hydromorphologique en cours d'eau. Agence française pour la Biodiversité, Paris, 176 p.
R CORE TEAM 2016. - R: a Language and Environment for Statistical Computing. R Foundation for Statistical Computing, Vienna. https://www.r-project.org/, dernière consultation le 20 juin 2020.

SAINT-JACQUeS N. \& RiChARD Y. 1998. — Développement d'un indice de qualité de la bande riveraine: application à la rivière Chaudière et mise en relation avec l'intégrité biotique du milieu aquatiqu, in MinisTère DE L'ENVIRONNEMENT ET DE LA FAUNE (éd.), Le bassin de la rivière Chaudière: l'état de l'écosystème aquatique. Direction des écosystèmes aquatiques, envirodoq EN980022, Québec: 6.1-6.41.

Tutin T. G., Heywood V. H. , Burges N. A., Valentine D. H., Walters S. M. \& WebB D. A. 2001. - Flora Europaea. Cambridge University Press, 2392 p.

Vargas-Luna A., Crosato A., Anders N., Hoitink A. J. F., KeEsstra S. D. \& UijtTeWAal W. S. J. 2018. — Morphodynamic effects of riparian vegetation growth after stream restoration. Earth Surface Processes and Landforms 43 (8): 1591-1607. https://doi.org/10.1002/esp.4338

WASSON J.-B. 2001. - Les questions de recherche posées par la Directive Cadre européenne sur I'Eau: problématique pour les eaux de surface continentales. Hydroécologie appliquée 13: 1-19 https://doi.org/10.1051/hydro:2001001 


\section{ANNEXES}

ANNEXE 1. - Abondances moyennes en 2018 des 22 espèces les plus fréquentes (> 20 occurrences), observées sur les 81 quadrats «Affluents» et les 156 quadrats «Rives» de la Sélune

\begin{tabular}{|c|c|c|}
\hline & $n=81 Q$ Affluents & n = 156 Q Rives \\
\hline Agrostis stolonifera L. & 0,02 & 0,2 \\
\hline Alnus glutinosa (L.) Gaertn. & 0,03 & 0,2 \\
\hline Atriplex patula L. & 0,2 & 0,06 \\
\hline Bidens tripartita L. & 0,1 & 0,3 \\
\hline Conyza canadensis (L.) Cronquist & 0,06 & 0,3 \\
\hline Epilobium tetragonum L. & 0,15 & 0,2 \\
\hline Gnaphalium uliginosum L. & 0,2 & 0,2 \\
\hline Holcus lanatus L. & 0,3 & 0,1 \\
\hline Juncus effusus L. & 1,2 & 1 \\
\hline Leersia oryzoides (L.) Sw. & 0,15 & 0,15 \\
\hline Lycopus europaeus L. & 0,75 & 0,4 \\
\hline Lythrum salicaria L. & 0,01 & 0,3 \\
\hline Myosotis scorpioides L. & 0,1 & 0,2 \\
\hline Phalaris arundinacea L. & 0,3 & 0,4 \\
\hline Persicaria hydropiper (L.) Spach & 1,8 & 0,5 \\
\hline Polygonum lapathifolium L. & 1,5 & 2 \\
\hline Polygonum persicaria L. & 0,2 & 0,3 \\
\hline Rorippa palustris (L.) Besser & 0,04 & 0,3 \\
\hline Salix atrocinerea Brot. & 0,3 & 0,3 \\
\hline Scrophularia auriculata L. & 0,1 & 0,1 \\
\hline Trifolium repens $\mathrm{L}$. & 0,01 & 0,3 \\
\hline Urtica dioica L. & 0,5 & 0,2 \\
\hline
\end{tabular}

ANNEXE 2. - Abondances moyennes en 2017 des 26 espèces les plus fréquentes (> 20 occurrences), observées sur les 48 quadrats «Affluents » et 126 quadrats «Rives» de la Sélune en 2017

\begin{tabular}{|c|c|c|}
\hline & $\mathrm{n}=48 \mathrm{Q}$ Affluents & $\mathrm{n}=126 \mathrm{Q}$ Rives \\
\hline Agrostis stolonifera L. & 0,06 & 0,33 \\
\hline Alnus glutinosa (L.) Gaertn. & 0,02 & 0,12 \\
\hline Cardamine flexuosa With. & 0,15 & $<0,01$ \\
\hline Conyza canadensis (L.) Cronquist & 0,02 & 0,12 \\
\hline Echinochloa crus-galli (L.) P.Beauv. & 0,15 & 0,18 \\
\hline Epilobium tetragonum L. & 0,13 & 0,02 \\
\hline Galium palustre L. & 0,09 & 0,07 \\
\hline Glyceria fluitans (L.) R.Br. & 0,25 & 0,06 \\
\hline Gnaphalium uliginosum L. & 0,46 & 0,08 \\
\hline Holcus lanatus L. & 0,19 & 0,14 \\
\hline Iris pseudacorus L. & 0,13 & 0,08 \\
\hline Juncus bufonius L. & 0,65 & 0,15 \\
\hline Juncus effusus L. & 1,67 & 0,57 \\
\hline Lycopus europaeus L. & 0,79 & 0,26 \\
\hline Lythrum salicaria $\mathrm{L}$. & 0,4 & 0,14 \\
\hline Myosotis laxa subsp. cespitosa (Schultz) Hyl. ex Nordh. & 0,13 & 0,05 \\
\hline Myosotis scorpioides L. & $<0,01$ & 0,14 \\
\hline Persicaria hydropiper (L.) Spach & $<0,01$ & 0,21 \\
\hline Polygonum lapathifolium L. & 0,9 & 1,75 \\
\hline Polygonum persicaria L. & 0,88 & 0,46 \\
\hline Ranunculus repens L. & 0,07 & 0,06 \\
\hline Rorippa palustris (L.) Besser & 0,13 & 0,22 \\
\hline Rumex conglomeratus Murray & 0,21 & 0,02 \\
\hline Salix atrocinerea Brot. & $<0,01$ & 0,06 \\
\hline Scrophularia auriculata L. & 0,27 & 0,13 \\
\hline Urtica dioica L. & 0,4 & 0,06 \\
\hline
\end{tabular}

